\title{
Effects of geometry on the convection with core-cooling
}

\author{
Yasuyuki Iwase and Satoru Honda \\ Department of Earth and Planetary Systems Science, Faculty of Science, Hiroshima University, \\ Kagamiyama 1-3-1, Higashi-Hiroshima 739-8526, Japan
}

(Received February 24, 1997; Revised April 16, 1998; Accepted April 17, 1998)

\begin{abstract}
We study the dynamical (three-dimensional box, axisymmetric and spherical shell geometry) and parameterized models of the mantle convection with the core-cooling. The viscosity is constant in space and dependent on the volume averaged mantle temperature. Core is treated as a hot bath. To understand the process of cooling, we use the 'local' Rayleigh $\left(R a_{l}\right)$ and Nusselt $\left(N u_{l}\right)$ numbers, which are defined in each thermal boundary layer. In the dynamical calculations, we check the various combinations of $R a_{l}$ and $N u_{l}$, and find that the local Rayleigh number either at the top or bottom surface may control both the top and bottom local Nusselt numbers. This result suggests that the core-cooling in this case may be controlled by the flow either at top or bottom boundary layer. The least-square-fitting of $N u_{l}-R a_{l}$ relationship shows that its power-law index is around 0.3 , despite of the different geometry. Comparing the thermal history calculated by the dynamical and parameterized models, we find that the parameterized convection theory based on the local $R a-N u$ relationship obtained by the dynamical calculation is useful for investigating the thermal history of the mantle and core. Applying the parameterized theory to the Earth, we find that the plausible Urey ratio is smaller than that obtained by the previous works which ignored the bottom thermal boundary layer.
\end{abstract}

\section{Introduction}

Mantle and core of the Earth and other terrestrial planets have been cooled by the convection of the mantle after the early differentiation of the core from the mantle. Studies of the convection of the mantle with core-cooling are important to understand the thermal evolution of the planets. Recently, because of the progress of the computer technologies, we can solve the dynamic equations of the convection directly by various numerical methods (see review of Schubert, 1992). Such studies show the important phenomena which may affect the thermal history of the Earth. For example, the studies of the thermal history of the Earth-like planets including the thermal coupling of the core and mantle show that the thermal boundary layer near the core-mantle boundary becomes weak as the cooling proceeds (Nakakuki, 1993; Arkani-Hamed, 1994; Honda and Iwase, 1996). The existence of the endothermic phase transition may induce a gigantic 'flushing' events in the Earth's history (Steinbach et al., 1993; Honda and Yuen, 1994; Yuen et al., 1995). However, since the properties of the mantle material and the 'initial' conditions of the early stages of the planets are highly unknown, the parameterized study (e.g., McKenzie and Weiss, 1975; Schubert et al., 1979; Davies, 1980; Stevenson et al., 1983; Christensen, 1985; Davies, 1993; Honda, 1995) of the thermal evolution of the mantle of the terrestrial planets is still useful. In the parameterized studies of thermal history of the mantle, the dynamics of the mantle flow is ignored and only mean thermal status of the mantle is calculated from the thermal input and output using the relationship among several

Copy right (C) The Society of Geomagnetism and Earth, Planetary and Space Sciences (SGEPSS); The Seismological Society of Japan; The Volcanological Society of Japan; The Geodetic Society of Japan; The Japanese Society for Planetary Sciences. parameters, such as Rayleigh number and Nusselt number. The parameterized studies are economical and easy to check many possible scenarios. Also, we may be able to infer the past status of the mantle from the present information on the mantle (Davies, 1980).

The parameterized convection theory is usually based on the power-law relationship between Rayleigh $(R a)$ and Nusselt $(N u)$ numbers (i.e., $\left.N u \propto R a^{\beta}\right)$, which is obtained by the studies of the steady state convection. In the steady state convection, $N u-R a$ relationship is the same at the top and bottom boundary layers and Stevenson et al. (1983) calculated the thermal evolution of the Mercury, Venus, Earth and Mars assuming $\beta=0.3$ at both top and bottom boundary layers. Christensen (1985) showed the relationship between Archaean thermal status of the Earth and $\beta$-value without considering the heat from the core (i.e., ignoring the bottom thermal boundary layer). However, in the cooling mantle, the mechanisms of the cooling may be different from that of the steady state convection (Davies, 1993). Davies (1993) considered four types of the heat removal mechanisms for the thermal boundary layer at the core-mantle boundary; (1) non-plume upwellings, in which the convection is driven by the local instabilities of both top and bottom boundary layers, (2) forced advection, or 'mantle wind', in which the convection is driven by the plate scale flow generated at the top boundary layer, (3) plume tails, in which the heat is carried through the established plume conduit or tail, and (4) new plume heads. Considering them, it may be better to treat each boundary layer separately in the study of the thermal evolution of the mantle convection. In our previous paper (Honda and Iwase, 1996), we showed that such a separate treatment is useful for a simple cooling model, although its parameterization is conceptually incomplete. 
Table 1. Parameters used for dynamical calculations.

\begin{tabular}{|c|c|c|c|c|c|}
\hline Geometry & Grid Point $^{(1)}\left(^{(2)}\right)$ & $K$ & $m$ & $\beta_{T}^{(3)}$ & $\beta_{B}^{(4)}$ \\
\hline 3D Box & $128 \times 128 \times 64(3: 3: 1)$ & 2 & 30 & 0.279 & 0.230 \\
\hline 3D Box & $128 \times 128 \times 64(3: 3: 1)$ & 10 & 30 & 0.354 & 0.367 \\
\hline 3D Box & $128 \times 128 \times 64(3: 3: 1)$ & 20 & 30 & 0.363 & none \\
\hline Axisymmetric & $128 \times 128(0.55)$ & 2 & 30 & 0.288 & 0.192 \\
\hline Axisymmetric & $128 \times 128(0.55)$ & 2 & 60 & 0.300 & 0.293 \\
\hline Axisymmetric ${ }^{(5)}$ & $128 \times 128(0.55)$ & 2.25 & 30 & 0.219 & 0.242 \\
\hline Axisymmetric & $128 \times 128(0.325)$ & 5 & 30 & 0.392 & 0.327 \\
\hline Axisymmetric & $128 \times 128(0.55)$ & 20 & 30 & 0.196 & 0.265 \\
\hline 3D Spherical Shell & $32 \times 32 \times 64(0.55)$ & 2 & 30 & 0.276 & 0.281 \\
\hline
\end{tabular}

${ }^{(1)} x \times y \times z$ (box model) or $r \times \theta(\times \phi)$ (spherical shell model). ${ }^{(2)}$ Aspect ratio (box model) or core size $\eta$ (spherical shell model). ${ }^{(3)} \beta_{T}$ for $N u_{T}-R a_{T}$ for box model and for $N u_{T}-R a_{B}$ for spherical shell model. ${ }^{(4)} \beta_{B}$ for $N u_{B}-R a_{T}$ for box model and for $N u_{B}-R a_{B}$ for spherical shell model. ${ }^{(5)}$ Start with the different initial condition from the other axisymmetric case.

In this paper, we extend our previous studies into threedimensional box and the spherical shell geometry models. In order to understand the core-cooling mode, we apply the 'local' Nusselt $\left(N u_{l}\right)$ and Rayleigh $\left(R a_{l}\right)$ numbers (Honda, 1996), which are more consistent parameters for local treatment than those used by Honda and Iwase (1996). We shall show the usefulness of $N u_{l}-R a_{l}$ relationship for the study of parameterized convection. We also reexamine the studies of the previous parameterized thermal history of the Earth.

\section{Numerical Method}

The models we use here is essentially the same as those described in Honda and Iwase (1996). We shall repeat here some of the important points.

\subsection{Fully dynamical calculation}

We assume the mantle as the infinite Prandtl number viscous fluid and adopt the Boussinesq approximation. Viscosity is constant in space and dependent on the volume averaged temperature of the mantle. Core is treated as a hot bath of constant temperature $T_{B}$ (temperature at bottom of the mantle). The equations to be solved are the equations of continuity, motion, and heat transfer of the mantle and core. Length, time, and temperature are normalized by thickness of the convection layer $d\left(=r_{T}-r_{B} ; r_{T}\right.$ is the radius of the top surface and $r_{B}$ is the radius of the core), thermal diffusion time $d^{2} / \kappa$ ( $\kappa$ is the thermal diffusivity), and temperature difference between the core and the surface of the mantle $\Delta T_{\text {ref }}\left(=T_{B}-T_{T} ; T_{T}\right.$ is the temperature at the surface) at time $t=0$, respectively. The non-dimensional expressions of equations of mass conservation, motion, and energy conservation for mantle and core, are respectively given by

$$
\left\{\begin{array}{l}
\nabla \cdot \boldsymbol{v}=0, \\
-\nabla P+v \nabla^{2} \boldsymbol{v}+R a_{r e f} T \boldsymbol{e}_{r}=\mathbf{0} \\
\frac{\partial T}{\partial t}+(\boldsymbol{v} \cdot \nabla) T=\nabla \cdot(\nabla T), \\
\frac{d T_{B}}{d t}=-K q_{B}
\end{array}\right.
$$

where $\boldsymbol{e}_{r}$ is the unit vector of the vertical direction (positive upward). $R a_{r e f}$ is the reference Rayleigh number defined by

$$
R a_{r e f}=\frac{\alpha g d^{3} \Delta T_{r e f}}{\kappa v_{r e f}}
$$

where $\alpha$ is the coefficient of the thermal expansion, $v_{\text {ref }}$ is the kinematic viscosity at $t=0, \kappa$ is the thermal diffusivity and $g$ is the acceleration of the gravity. The normalized viscosity of the mantle $v$ is assumed to depend only on the volume averaged temperature of the mantle $T_{m}$, which is given by

$$
v=\left(T_{m} / T_{r e f}\right)^{-m}
$$

where $T_{\text {ref }}$ is the averaged temperature at $t=0$. Power-law index $m$ is a constant and is around 30 for the mantle of the Earth (Davies, 1980). Other material properties are assumed to be constant. $K$ is the non-dimensional number related to the cooling of the core and is given by

$$
K=\frac{3 \rho_{m} C_{m} d}{\rho_{c} C_{c} r_{B}}
$$

where $\rho_{m}, C_{m}, \rho_{c}$, and $C_{c}$ is the density and the specific heat of the mantle and core, respectively (Steinbach et al., 1993). For the Earth, $K$ is around 2. Boundary conditions are freeslip and constant temperature on top and bottom boundaries.

The basic equations are solved by the finite (control) volume method (Patankar, 1980). The details of the methodology are described in Tackley (1993) for box model and Iwase 
Axisymmetric

$\mathrm{K}=2, \mathrm{~m}=30, \eta=0.55$
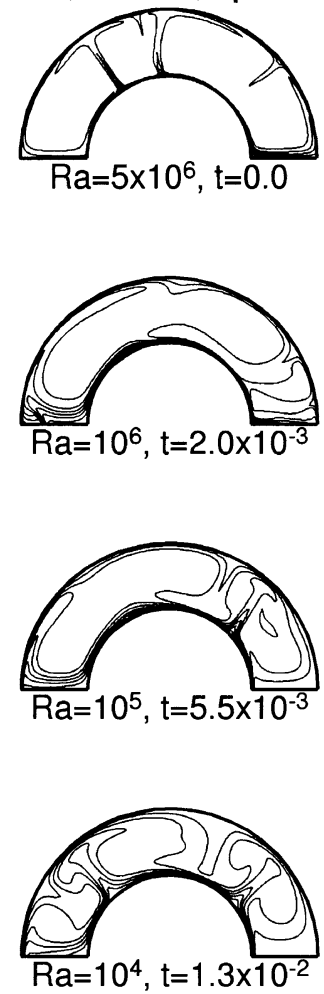
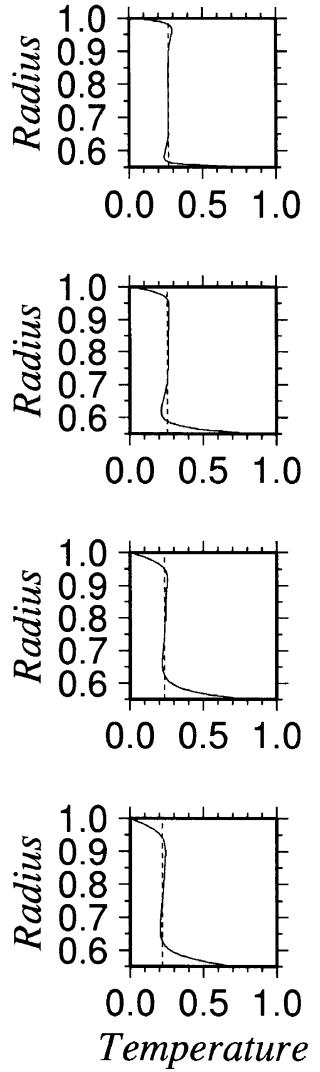

Full 3D

$\mathrm{K}=2, \mathrm{~m}=30, \eta=0.55$
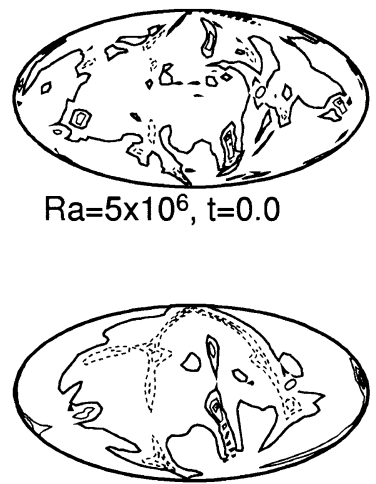

$\mathrm{Ra}=10^{6}, \mathrm{t}=2.3 \times 10^{-3}$

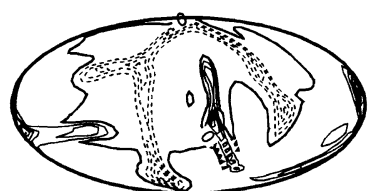

$\mathrm{Ra}=9.7 \times 10^{4}, \mathrm{t}=4.0 \times 10^{-3}$

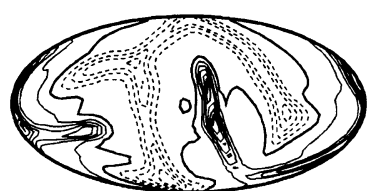

$\mathrm{Ra}=10^{4}, \mathrm{t}=6.5 \times 10^{-3}$
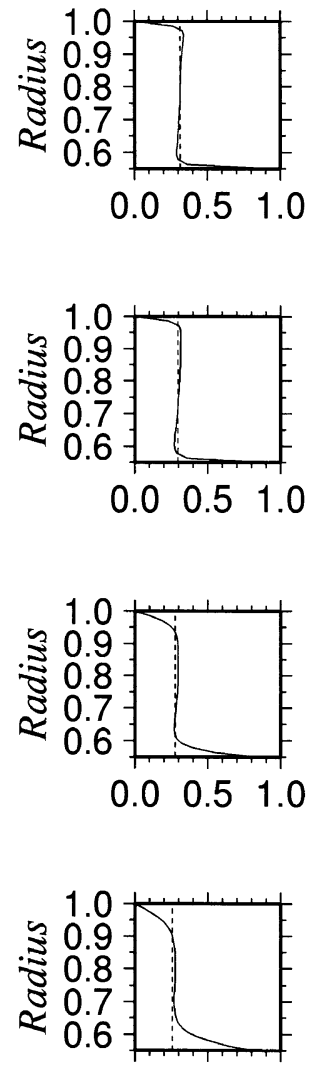

$0.0 \quad 0.51 .0$

Temperature

Fig. 1. Flow pattern (left) and horizontally averaged temperature profile (right) for axisymmetric model $(\eta=0.55, K=2$ and $m=30$ ) at $R a=5 \times 10^{6}$ (initial condition; first panel), $\sim 10^{6}$ (second panel), $\sim 10^{5}$ (third panel) and $\sim 10^{4}$ (last panel). Temperature is normalized by the initial core temperature. Contour interval is 0.1 non-dimensional unit. Dashed lines in vertical temperature profile (right figures) show the globally averaged temperature $\left(T_{m}\right)$.

(1996) for spherical shell models. Axisymmetric model is calculated by setting the physical parameters, such as the velocity, temperature, and pressure, to be independent of longitudinal coordinate and the longitudinal velocity to be zero.

\subsection{Parameterized calculation}

For the calculation of the parameterized convection, we consider the simple energy balance of the core and the mantle. Non-dimensional expressions of these equations are given by

$$
\left\{\begin{aligned}
\frac{d T_{m}}{d t} & =\frac{3}{1+\eta+\eta^{2}}\left(\eta^{2} q_{B}-q_{T}\right)+H, \\
\frac{d T_{B}}{d t} & =-K q_{B}
\end{aligned}\right.
$$

where $\eta$ is the ratio of the radius of the core to that of the surface of the mantle (i.e., $\eta=r_{B} / r_{T}$ ) and $H$ is the internal heat generation. Note that $\eta=1$ corresponds to the cartesian cases and $\eta=0.55$ corresponds to the Earth's mantle. The heat fluxes at the top $q_{T}$ and bottom $q_{B}$ are obtained from the $N u$ - $R a$ relationship. This point is discussed in the later section. The time integration of the above equations is executed backward using the forth-order Runge-Kutta method.

\section{Local Parameterization}

To treat the boundary layers separately, we introduce the 'local' Rayleigh $\left(R a_{l}\right)$ and Nusselt $\left(N u_{l}\right)$ numbers (Honda, 1996). Here, we shall repeat their arguments to clarify the following discussions. They are defined separately at the top and bottom thermal boundary layers as

$$
\left\{\begin{aligned}
R a_{l} & \equiv \frac{\alpha g \Delta T_{l} d^{3}}{\kappa v} \\
N u_{l} & \equiv \frac{q_{l}}{k \Delta T_{l} / d}
\end{aligned}\right.
$$

where $\Delta T_{l}$ and $q_{l}$ are the temperature drop in the top or bottom thermal boundary layer and the heat flux through the corresponding boundary layers, respectively. The suffix $l$ implies the 'local' value ( $l$ is either $T$ : top or $B$ : bottom). Honda (1996) showed that $R a_{l}$ and $N u_{l}$ are convenient parameters to interpret the results of the steady cartesian con- 
Nu (local) vs. Ra (local)
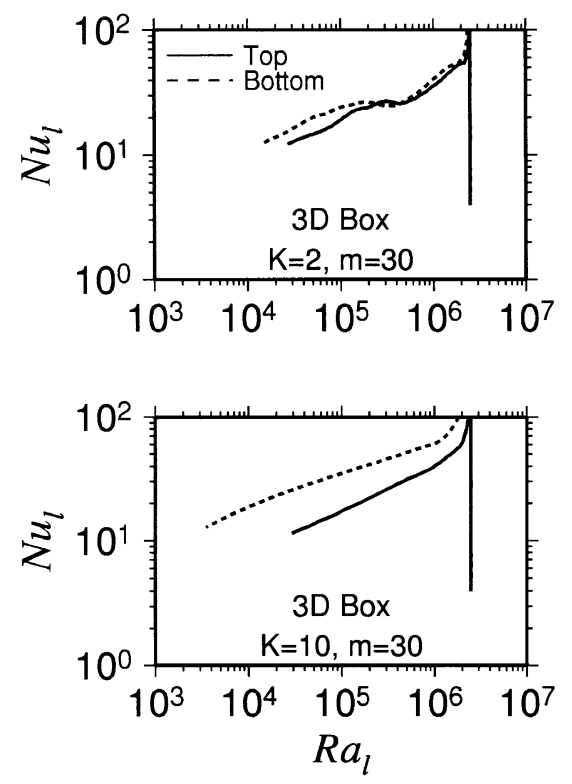

Nu (local) vs. Ra (local)
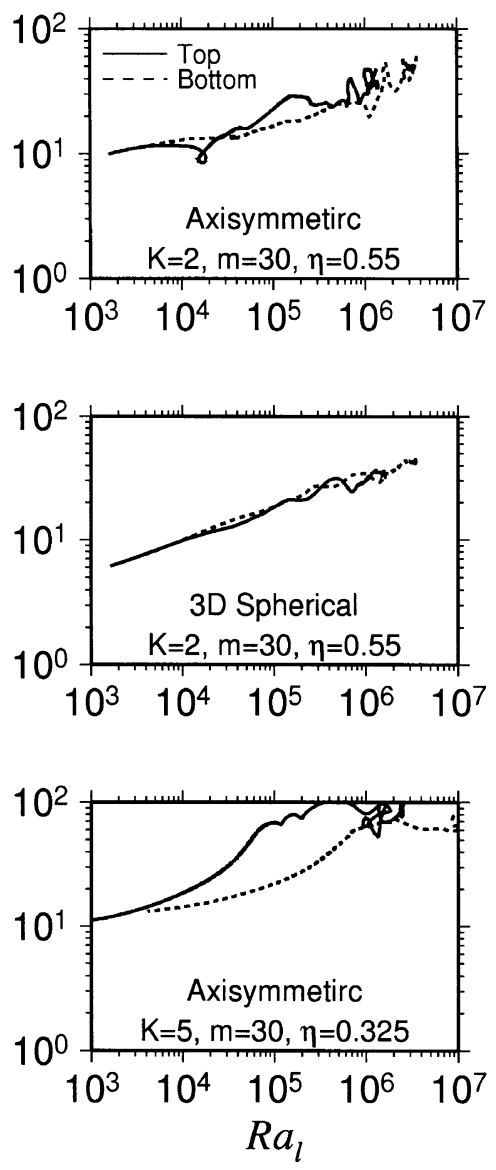

Fig. 3. Relationship between temporal local Nusselt $\left(N u_{l}\right)$ and Rayleigh $\left(R a_{l}\right)$ numbers $\left(R a_{T}\right.$ vs. $N u_{T} ;$ solid line and $R a_{B}$ vs. $N u_{B}$; dashed line).

vection with a variable viscosity.

If the thermal boundary layer thickness is controlled by its own gravitational instability (Howard, 1966), we may write such condition as

$$
\frac{\alpha g \Delta T_{l} \delta_{l}^{3}}{\kappa \nu}=R a_{c l}
$$

where $\delta_{l}$ is the thickness of the thermal boundary layer and $R a_{c l}$ is a constant. Since

$$
q_{l} \sim k \frac{\Delta T_{l}}{\delta_{l}},
$$

we may obtain

$$
q_{l} \sim\left(\frac{R a_{l}}{R a_{c l}}\right)^{1 / 3} k \frac{\Delta T_{l}}{d} .
$$

This implies

$$
N u_{l} \sim\left(\frac{R a_{l}}{R a_{c l}}\right)^{1 / 3} .
$$

Generalizing this result, we may write the relationship between $N u_{l}$ and $R a_{l}$ as

$$
\left\{\begin{array}{l}
N u_{T}=a R a_{T}^{\beta} \\
N u_{B}=a R a_{B}^{\beta}
\end{array}\right.
$$

where $\beta$ is a constant supposed to be close to $1 / 3$. Implicit assumption in Eq. (11) is that $R a_{c l}$ is constant at bottom and top boundary layers.

Davies (1993) showed that, if the core is cooled by the flow induced by the plate scale flow (i.e., forced convection),

$$
q_{B}=\gamma \frac{\Delta T_{B}}{\Delta T_{T}} q_{T}
$$

where $\gamma$ is a constant (Eq. (9) of Davies, 1993). Since he assumed that the thermal conductivity is constant throughout the mantle, this relationship may be rewritten as

$$
N u_{B}=\gamma N u_{T}
$$

This may imply that both local Nusselt numbers may be controlled by either $R a_{T}$ or $R a_{B}$ (i.e., single $R a_{l}$ ).

\section{Results and Discussions}

\subsection{Dynamical calculation}

We carry out the fully dynamical calculations of threedimensional box and the spherical shell models. The conditions and the parameters $K$ and $m$ are listed in Table 1 . The model with $K=2$ and $m=30$ roughly equivalent to the real Earth. All the calculations start with the initial Rayleigh number of $R a_{r e f}=5 \times 10^{6}$. For the three-dimensional box 
Nu (local) vs. Ra (top)
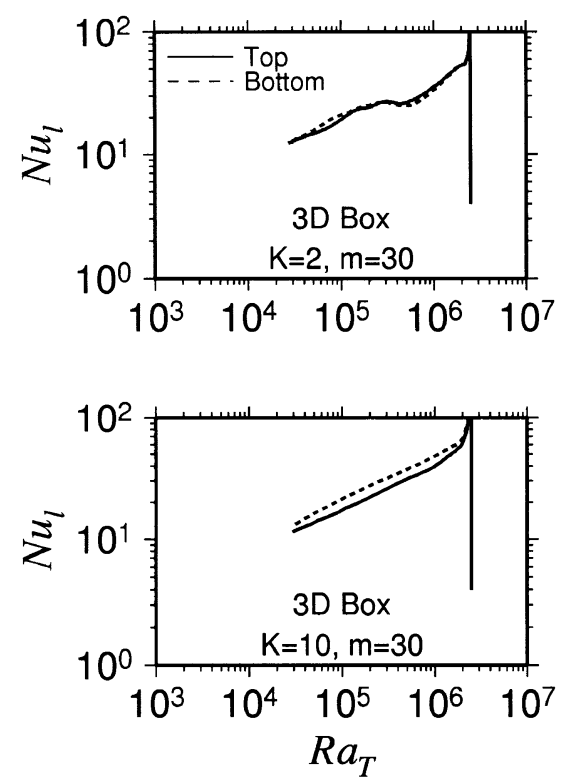

Nu (local) vs. Ra (bottom)
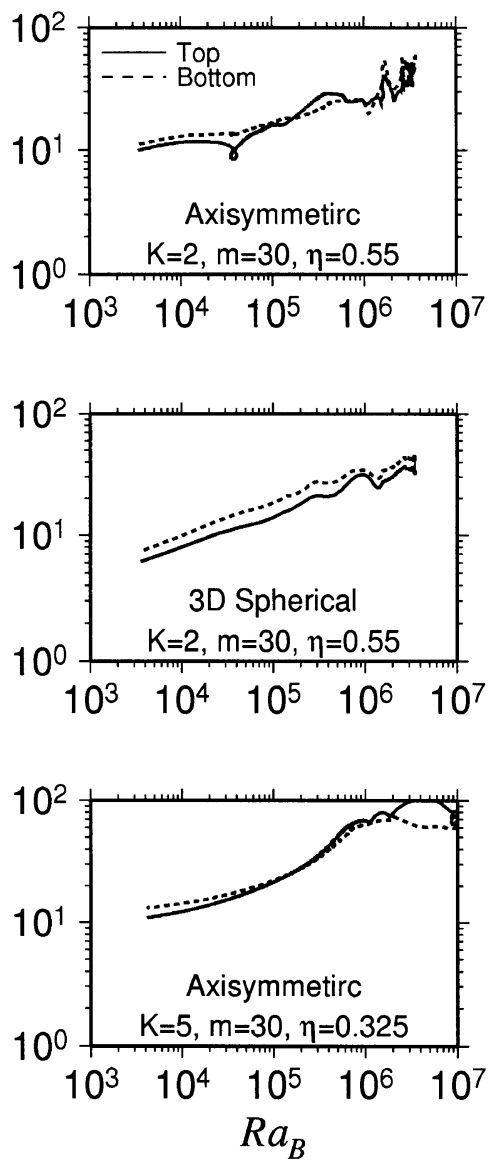

Fig. 4. Relationship between temporal local Nusselt $\left(N u_{l}\right)$ and top Rayleigh number ( $\left.R a_{T}\right)$ for box models (left) and bottom Rayleigh number $\left(R a_{B}\right)$ for spherical shell models (right).

model, the number of control volumes are $128 \times 128$ for horizontal and 64 for vertical directions. For the axisymmetric and full three-dimensional models, the number of the control volumes are $128 \times 128(r \times \theta)$ and $32 \times 32 \times 64(r \times \theta \times \phi)$, respectively. At the high Rayleigh number $\left(R a>10^{6}\right)$, this mesh configuration may not be enough. However, as we will show, the results are mainly controlled by the Rayleigh number below $10^{6}$. Thus, the general conclusion obtained in this study is considered to be valid, at least, up to $R a \sim 10^{6}$.

Figures 1 and 2 show the flow pattern and the horizontally averaged temperature for the Earth-like model $(K=2$ and $m=30$ ) for the axisymmetric (Fig. 1) and full threedimensional spherical shell (Fig. 2) geometry. As the mantle and core cool down, temperature drop in the bottom thermal boundary layer gradually decreases. This is also found in our previous two-dimensional box models (Honda and Iwase, 1996).

To define $N u_{l}$ and $R a_{l}$, we assume that the temperature drops of each thermal boundary layers are $\Delta T_{T}=T_{m}-T_{T}$ for top and $\Delta T_{B}=T_{B}-T_{m}$ for bottom thermal boundary layer.

Figure 3 shows the temporal relationship of $N u_{T}-R a_{T}$ and $N u_{B}-R a_{B}$. As the core-cooling constant $K$ becomes large (i.e., the rate of cooling becomes large), the difference between $N u_{T}-R a_{T}$ and $N u_{B}-R a_{B}$ becomes large. We note that the gradient of $N u_{T}-R a_{T}$ and $N u_{B}-R a_{B}$ relationships is different from each other.

The decrease in the mean temperature by the cooling is much smaller than that in the temperature of the core as shown Figs. 1 and 2 (see, also Honda and Iwase, 1996). Thus, since the temperature drop within the top boundary layer is larger than that of the bottom (i.e., $\Delta T_{T}>\Delta T_{B}$ ) for the cartesian geometry, the top local Rayleigh number is larger than bottom local Rayleigh number $\left(R a_{T}>R a_{B}\right.$, see Eq. (11)). However, for the spherical shell geometry, the bottom Rayleigh number is still larger than the top Rayleigh number $\left(R a_{B}>R a_{T}\right)$. It may be natural to think that the flow is controlled by the instability at the boundary layer which has larger local Rayleigh number (Iwase and Honda, 1997). Thus, we consider that $N u_{l}$ may be controlled by $R a_{T}$ for the box models and by $R a_{B}$ for the spherical shell models.

In Fig. 4, we show the temporal relationship between local Nusselt numbers (both $N u_{T}$ and $N u_{B}$ ) and top Rayleigh number $\left(R a_{T}\right)$ for the box models and bottom Rayleigh number $\left(R a_{B}\right)$ for the spherical shell models. The difference between the $N u_{l}-R a_{T}$ (box models) or $N u_{l}-R a_{B}$ (spherical shell models) relationships for top and bottom boundary lay- 

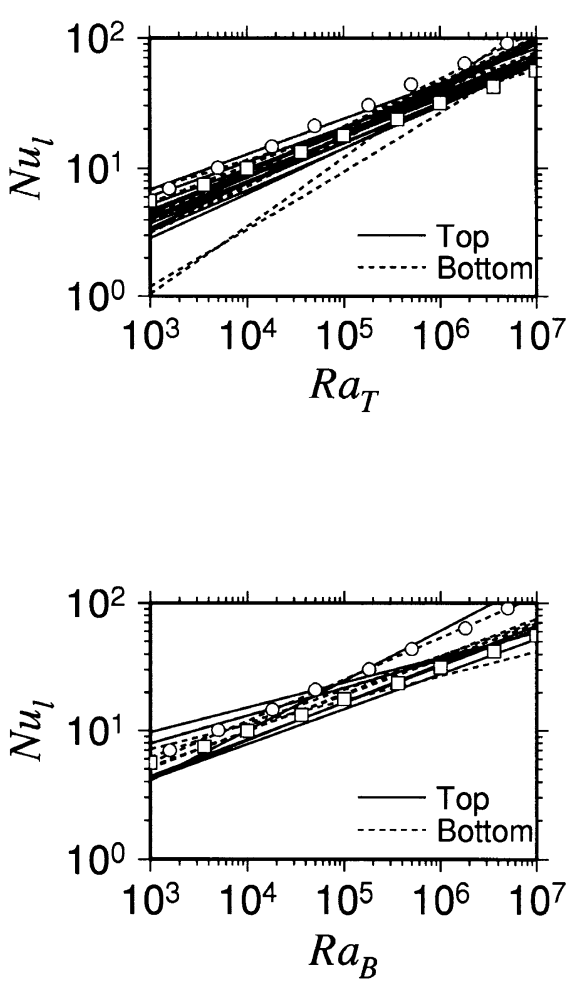

Fig. 5. Summary of the relationship between local Nusselt $\left(N u_{l}\right)$ and Rayleigh $\left(R a_{l}\right)$ numbers. Least square fitting lines of temporal $N u_{l}$ and $R a_{l}$ are plotted. (a) $N u_{l}-R a_{T}$ relationship for two- (Honda and Iwase, 1996) and three-dimensional box models. (b) $N u_{l}-R a_{B}$ relationship for spherical shell models. $N u_{l}-R a_{l}$ relationship for the steady state constant viscosity case of two-dimensional box case (Christensen, 1984) is plotted by circles and that for spherical shell case (Iwase and Honda, 1997) is plotted by squares.

ers in Fig. 4 becomes smaller than that of $N u_{l}-R a_{l}$ in Fig. 3, especially for the fast cooling case. This implies that $N u_{l}$ is controlled by a single local Rayleigh number and suggests that the core-cooling is controlled mainly by the flow either at the top or bottom.

The relationships of $N u_{l}-R a_{T}$ for the box and $N u_{l}-R a_{B}$ for the spherical shell models are summarized in Fig. 5. They are obtained by the least-square-fitting of previous results. Since results at $R a>10^{6}$ are strongly affected by the initial condition, we use the results only with $R a<10^{6}$ for this discussion. The results of the two-dimensional box case (Table 2 of Honda and Iwase, 1996) are also included in Fig. 5(a). We show the $N u_{l}-R a_{l}$ relationship for the steady constant viscosity convection with two-dimensional box geometry calculated by the results of Christensen (1984) (white circles) and the spherical shell (Iwase and Honda, 1997) geometry (white rectangles). The power-law index of $N u_{l}-R a_{T}$ or $N u_{l}-R a_{B}$ relationship, $\beta$-values are around 0.3 (Table 1) for all the calculations except for the cases with large $K$, in which because of rapid cooling, the bottom boundary layer almost disappear and it results in the breakdown of the $N u_{l^{-}}$ $R a_{l}$ relationship (Honda and Iwase, 1996). Although there exits a difference in the $N u_{l}-R a_{l}$ relationship for the steady state convection of spherical shell $(\beta \sim 1 / 4)$ and box geometry $(\beta \sim 1 / 3)$ (Iwase, 1996; Ratcliff et al., 1996), our
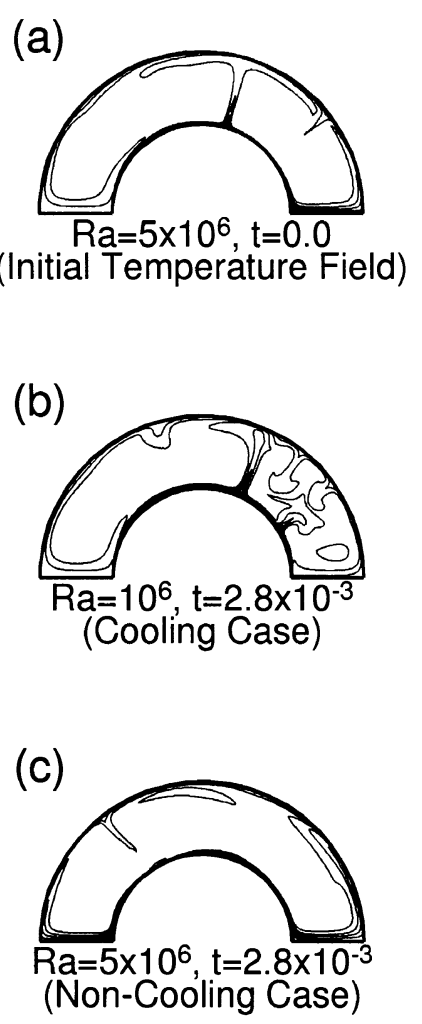

Fig. 6. Comparison of flow pattern for core-cooling and no-cooling cases. Axisymmetric model is used $(\eta=0.55)$. (a) Initial condition $(t=0)$, (b) core-cooling case $(K=2.25$ and $m=30)$, at $t=2.8 \times 10^{-3}$ and (c) non-cooling case at $t=2.8 \times 10^{-3}$.

results suggest that there is no practical difference in $\beta$ for the convection with cooling.

In this study, the internal heating is ignored $(H=0)$. However, we already found that the heat transport efficiency for the internal heating case does not show much difference from that of the cases with bottom heating only (see also Honda and Iwase, 1996). If we include the internal heating, the lower thermal boundary layer would become weaker. Thus, the cooling by the flow at the top boundary layer would be promoted by the internal heating.

For the Earth model with the axisymmetric geometry, the flow pattern of mantle rapidly changes at high Rayleigh number (Fig. 1). However, we find that in another case, the major flow pattern is preserved as shown in Fig. 6(a) and (b), that is, the upwelling near the equator and downwelling at the pole. To check if this is affected by the cooling, we start the calculation with the same initial condition (i.e., Fig. 6(a)) without cooling. The flow pattern changes completely within the same time interval as the cooling continues (Fig. 6(c)). Although this result is obtained for axisymmetric model with low Rayleigh number $\left(R a<5 \times 10^{6}\right)$ and the change in the flow pattern may be also affected by the initial condition, it may suggest that the past mantle flow pattern of the Earth may be 'frozen' in some situations.

\subsection{Parameterized calculation}

In this section, we discuss the usefulness of the $N u_{l^{-}}$ $R a_{l}$ relationship obtained by the dynamical calculations for the parameterized convection theory. In the preceding sec- 

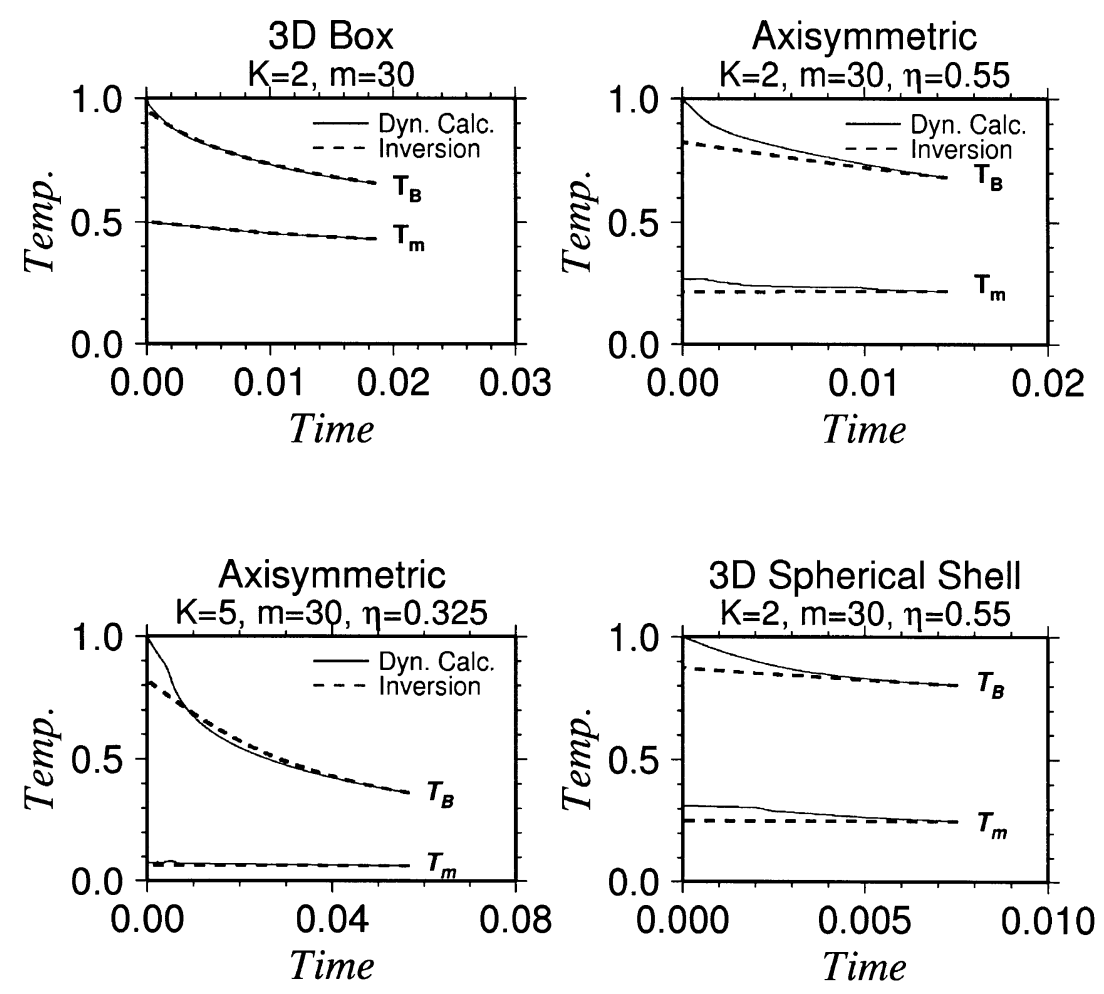

Fig. 7. Time evolution of the core temperature $\left(T_{B}\right)$ and the mean mantle temperature $\left(T_{m}\right)$. Results of the dynamical calculation are plotted by solid lines and those of the parameterized calculation are plotted by dashed lines.

tion, we show that the core-cooling may be controlled by either top or bottom thermal boundary layer. Thus, the nondimensional heat fluxes at the top $\left(q_{T}\right)$ and bottom $\left(q_{B}\right)$ may be written by

$$
\left\{\begin{array}{l}
q_{T}=N u_{T} \Delta T_{T}=a_{T} R a_{l}^{\beta_{T}} \Delta T_{T}, \\
q_{B}=N u_{B} \Delta T_{B}=a_{B} R a_{l}^{\beta_{B}} \Delta T_{B}
\end{array}\right.
$$

$\left(\beta_{T} \approx \beta_{B}\right)$ where $l$ is either $T$ for the box and $B$ for the spherical shell models, i.e., larger local Rayleigh number is used. The parameters $a_{l}$ and $\beta_{l}$ are determined by the leastsquare-fitting of the $N u_{l}-R a_{l}$ relationship obtained by the dynamical calculation (see Fig. 5 and Table 1). The initial core and mean mantle temperatures of this calculation are set to be the final temperatures of the dynamical calculation. The internal heating is ignored ( $H=0$ in Eqs. (5)) to be consistent with the dynamical calculations.

The changes of the mean mantle $\left(T_{m}\right)$ and core $\left(T_{B}\right)$ temperatures with time for both dynamical and parameterized calculations are shown in Fig. 7. Both results show fairly good agreements. The difference of the results between dynamical and parameterized calculations in the spherical shell models may be due to simple least-square-fitting to obtain $N u_{l}-R a_{B}$ relationship. The $\beta$-values in spherical shell models are larger at high Rayleigh number region (Fig. 4). In the steady state convection, $\beta$ shows the smaller value $(\sim 0.25)$ at lower Rayleigh number region, since the flow pattern is strongly affected by the spherical shell geometry (Iwase and Honda, 1997). For the cooling case, $\beta$-value may become smaller at lower Rayleigh number region. If we take larger $\beta$-value (say, $\sim 0.05$ larger), the difference in results between dynamical and parameterized calculation becomes much smaller. Thus, we conclude that the parameterized model based on the relationship of $N u_{l}-R a_{l}$ may be valid for the study of the thermal history of the mantle and core.

\subsection{Application to the thermal history of the Earth}

In this section, we apply the parameterized convection theory to the thermal history of the mantle and core of the Earth based on our results. The equations to be solved are essentially identical to Eqs. (5). They are integrated backward in time. The thermal properties of the present status of the mantle and core of the Earth are taken as the initial values of our calculation (see below).

In order to compare the results with those of the previous works (Christensen, 1985; Honda, 1995) as discussed below, the Arrhenius type viscosity law is used, i.e.,

$$
v=v_{\text {ref }} \exp \left(T_{a} / T_{m}\right)
$$

where $T_{a}$ is the activation temperature $\left(T_{a}=63000 \mathrm{~K}\right)$ (Christensen, 1985). Note that plausible Urey ratio (see below) using this viscosity law is found to be almost the same with the case if we use the power-law viscosity, Eq. (3).

The heat generation by the radioactive isotopes, ${ }^{238} \mathrm{U},{ }^{235} \mathrm{U}$, ${ }^{232} \mathrm{Th}$ and ${ }^{40} \mathrm{~K}$ is taken into account. Their present amounts and decay constants are taken from Turcotte and Schubert (1982). We assume that the cooling of the core is controlled by the instability at the bottom thermal boundary layer. We also assume that the proportional constant between the top and bottom local Nusselt number $(\gamma)$ is unity, by considering our results. These assumptions imply $\beta_{T}=\beta_{B}=\beta$ and 
(a)

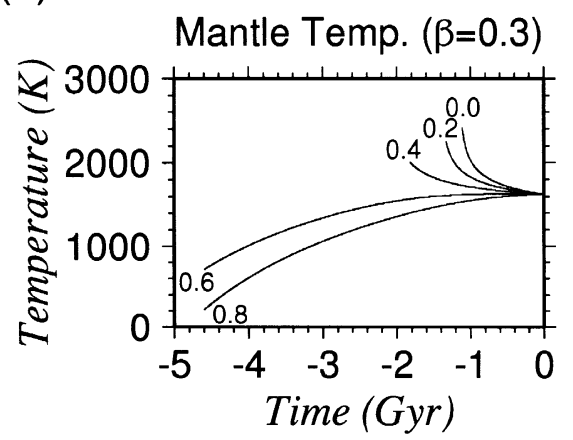

(b)

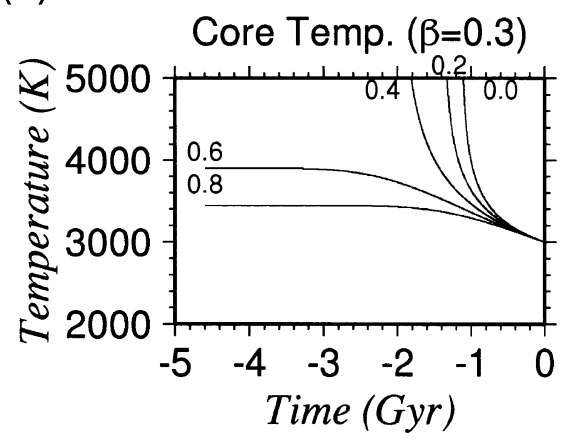

Fig. 8. (a) Mean mantle temperature and (b) core temperature with $\beta=0.3$ and $\gamma=1.0$ for various Urey ratio (numerals).

$a_{T}=a_{B}$ in Eq. (14). If we take $T_{T}=300 \mathrm{~K}, T_{m}=1623 \mathrm{~K}$ (Christensen, 1985) and $T_{B}=3000 \mathrm{~K}$ (Stevenson et al., 1983) for the present potential temperature of the mantle and core, the bottom Rayleigh number is higher than the top Rayleigh number, assuming that the gravity, the coefficient of the thermal expansion, the viscosity and the thermal conductivity are constant. Since $R a_{B}$ is found to be always larger than $R a_{T}$, we use $R a_{B}$ as the controlling parameter. We take the present values of the surface heat flow and the effective specific heat of the whole Earth to be $3.7 \times 10^{13} \mathrm{~W}$ and $7 \times 10^{27} \mathrm{~J} \mathrm{~kg}^{-1}$, respectively (Christensen, 1985). We assume that the heat capacity of the core is one-fifth of that of the mantle (Stacey, 1981).

The free parameters are only $\beta$ and the Urey ratio, which is the ratio of radiogenic heat production to the heat loss at the surface. We show the temperature of the mantle and core in Fig. 8 for $\beta=0.3$. As has been shown by previous workers (Davies, 1980; Christensen, 1985; Honda, 1995), the thermal runaway of the mantle can be observed. The Urey ratio around 0.5 gives a reasonable temperature of the mantle and core at $\sim 4.5 \mathrm{Ga}$. Christensen (1985) (also see Honda, 1995) studied the similar model, assuming that the rate of cooling of the core is equal to that of the mantle. This assumption implies that the heat capacity of the core is so small that the thermal inertia of the core or the bottom thermal boundary layer can be ignored. As a result, they found that the higher Urey ratio $(>0.8)$ is preferable for $\beta=0.3$. However, as our calculation shows, this conclusion should be revised by taking into account the effects of bottom boundary layer.

We also investigate the thermal history of the Earth assuming that the top local Nusselt number is controlled by the top local Rayleigh number and the bottom local Nusselt number is controlled by the bottom local Rayleigh number. The results are very similar to those assuming that the cooling is controlled only by the bottom local Rayleigh number. As discussed in the above, if the ratio of the core to the whole planet is close to the Earth, the difference of the cooling by these modes is not so obvious. However, the cooling by the flow controlled by either top or bottom thermal boundary layer may play a significant role in thermal evolution for the planets and satellites whose core size is smaller than that of the Earth $(\eta<0.55)$ or when their core has not grown to the size comparable to the Earth.

\section{Conclusions and Discussions}

We conduct the numerical simulation of the mantle convection with core-cooling using a simple model. The geometry of the model is either three-dimensional box, or axisymmetric and three-dimensional spherical shell. We assume that the viscosity is constant in space and changes as a function of the mean mantle temperature. We find the better local Nusselt and Rayleigh numbers relationship, when $R a_{l}$ is either $R a_{B}$ or $R a_{T}$. This suggests that the core may be cooled forcibly by the instability of either top or bottom boundary layer. The thermal history of the mantle and core calculated using the parameterized convection theory based on the $\mathrm{Ra}_{l^{-}}$ $N u_{l}$ relationship shows a good agreement with that obtained by the dynamical calculations. The thermal history of the Earth is calculated using the parameterized theory assuming that the bottom Rayleigh number controls both top and bottom Nusselt numbers. The appropriate Urey ratio is found to be smaller than that obtained by the model which neglects the effects of bottom boundary layer.

The Rayleigh number of the present Earth is larger than that used in this study and we neglect the effects of phase transitions, spatial viscosity variation and time-dependent internal heat generation. Although we have already studied the case including internal heating for two-dimensional box model (Honda and Iwase, 1996), further consideration is necessary for the spherical shell geometry. Heat transport may be affected by the cooling rate, though the effect of the cooling rate $(K)$ is not clearly shown in this study. The cooling of the core may also be influenced by the plume heads or tails (Davies, 1993), which are related to the temperaturedependent viscosity. Van den Berg and Yuen (1995) pointed out that the effects of the rheological transition on the dynamical evolution of the Earth's mantle must be taken into account in realistic dynamical models of a cooling Earth. The inclusion of these factors for the convection with corecooling should be done in the future.

Acknowledgments. We thank P. J. Tackley for using his code. We also appreciate Dr. Ogawa and an anonymous reviewer for their comments. This study was partly supported by the Japan-U.S. Cooperative Science Program. Y.I. was supported by the JSPS Research Fellowship. 


\section{References}

Arkani-Hamed, J., Effects of the core cooling on the internal dynamics and thermal evolution of terrestrial planets, J. Geophys. Res., 99, 12109$12119,1994$.

Christensen, U. R., Heat transport by variable viscosity convection and implications for the Earth's thermal evolution, Phys. Earth Planet. Inter., 35, 264-282, 1984.

Christensen, U. R., Thermal evolution models for the earth, J. Geophys. Res., 90, 2995-3007, 1985.

Davies, G. F., Thermal histories of convective Earth models and constraints on radiogenic heat production in the Earth, J. Geophys. Res., 85, 25172530, 1980.

Davies, G. F., Cooling the core and mantle by plume and plate flows, Geophys. J. Int., 115, 132-146, 1993.

Honda, S., A simple parameterized model of Earth's thermal history with the transition from layered to whole mantle convection, Earth Planet. Sci. Lett., 131, 357-369, 1995.

Honda, S., Local Rayleigh and Nusselt numbers for cartesian convection with temperature-dependent viscosity, Geophys. Res. Lett., 23, 2445 2448, 1996.

Honda, S. and Y. Iwase, Comparison of the dynamical and parameterized models of mantle convection including core-cooling, Earth Planet. Sci. Lett., 139, 133-146, 1996.

Honda, S. and D. A. Yuen, Cooling model of mantle convection with phase changes: effects of aspect ratio and initial conditions, J. Phys. Earth, 42, 165-186, 1994.

Howard, L. N., Convection at high Rayleigh number, in Proc. 11th Int. Cong. Appl. Math., edited by H. Görtler, pp. 1109-1115, Springer-Verlag, New York, 1966.

Iwase, Y., Three-dimensional infinite Prandtl number convection in a spherical shell with temperature-dependent viscosity, J. Geomag. Geoelectr. 48, 1499-1514, 1996.

Iwase, Y. and S. Honda, An interpretation of the Nusselt-Rayleigh number relationship for convection in a spherical shell, Geophys. J. Int., 130, 801-804, 1997.

McKenzie, D. P. and N. O. Weiss, Speculations on the thermal and tectonic history of the earth, Geophys. J. R. Astron. Soc., 42, 131-174, 1975.

Nakakuki, T., Studies of convection in the mantle with the phase and the chemical boundaries by numerical simulations, Ph.D. Thesis, Ocean Research Institute, Univ. Tokyo, 1993.

Patankar, S. V., Numerical Heat Transfer and Fluid Flow, 197pp., Hemisphere Pub. Corp., New York, 1980.

Ratcliff, J. T., G. Schubert, and A. Zebib, Steady tetrahedral and cubic patterns of spherical-shell convection with temperature-dependent viscosity, J. Geophys. Res., 101, 25473-25484, 1996.

Schubert, G., Numerical models of mantle convection, Annu. Rev. Fluid Mech., 24, 359-394, 1992.

Schubert, G., P. Cassen, and R. E. Young, Subsolidus convective cooling histories of terrestrial planets, Icarus, 38, 192-211, 1979.

Stacey, F. D., Cooling of the earth-A constraint on paleotectonic hypotheses, in Evolution of the Earth, edited by R. J. O'Connell and W. S. Fyfe, Geodyn. Ser., vol. 5, pp. 272-276, AGU, Washington, 1981.

Steinbach, V., D. A. Yuen, and W. Zhao, Instabilities from phase transitions and the timescales of mantle convection, Geophys. Res. Lett., 20, 1119 1122,1993

Stevenson, D. J., T. Spohn, and G. Schubert, Magnetism and thermal evolution of the terrestrial planets, Icarus, 54, 466-489, 1983.

Tackley, P. J., Effects of strongly temperature-dependent viscosity on timedependent, three-dimensional models of mantle convection, Geophys. Res. Lett., 20, 2187-2190, 1993.

Turcotte, D. L. and G. Schubert, Geodynamics, 450pp., John Wiley, New York, 1982.

van den Berg, A. P. and D. A. Yuen, Convectively induced transition in mantle rheological behavior, Geophys. Res. Lett., 22, 1549-1552, 1995.

Yuen, D. A., S. Balachandar, V. C. Steinbach, S. Honda, D. M. Reuteler, J. J. Smedsmo, and G. S. Lauer, Non-equilibrium effects of core-cooling and time-dependent internal heating on mantle flush events, Nonlinear Process. Geophys., 2, 206-221, 1995.

Y. Iwase (e-mail: iwase@sci.hiroshima-u.ac.jp) and S. Honda 